\title{
Time-dependent density-functional theory/localized density matrix method for dynamic hyperpolarizability
}

\author{
Fan Wang, Chi Yung Yam, and GuanHua Chen ${ }^{\text {a) }}$ \\ Department of Chemistry, The University of Hong Kong, Pokfulam Road, Hong Kong
}

(Received 3 April 2007; accepted 9 May 2007; published online 22 June 2007)

\begin{abstract}
Time-dependent density-functional theory/localized density matrix method (TDDFT/LDM) was developed to calculate the excited state energy, absorption spectrum and dynamic polarizability. In the present work we generalize it to calculate the dynamic hyperpolarizabilities in both time and frequency domains. We show that in the frequency domain the $2 n+1$ rule can be derived readily and the dynamic hyperpolarizabilities are thus calculated efficiently. Although the time-domain TDDFT/ LDM is time consuming, its implementation is straightforward because the evaluation of the derivatives of exchange-correlation potential with respect to electron density is avoided. Moreover, the time-domain method can be used to simulate higher order response which is very difficult to be calculated with the frequency-domain method. () 2007 American Institute of Physics.
\end{abstract}

[DOI: $10.1063 / 1.2746034]$

\section{INTRODUCTION}

The nonlinear optical effects of molecules have important application in optical-electronic devices. Some nonlinear optical effects, such as the second harmonic generation (SHG) or frequency doubling, the optical rectification (OR), and the electro-optic Pöckels effect (EOPE), are the secondorder response of a molecule due to external electric fields and are characterized by dynamic first hyperpolarizabilities. In addition, many other properties such as the two-photon absorption cross sections and the properties of the excited states can also be determined through the poles of the dynamic first hyperpolarizabilities. Several theoretical methods have been developed to calculate the dynamic hyperpolarizabilities. ${ }^{1-5}$ Recently, time-dependent densityfunctional theory ${ }^{6}$ (TDDFT) is formulated to calculate the dynamic hyperpolarizability. ${ }^{7-13}$ TDDFT with local density approximation $^{14,15}$ (LDA) usually overestimates the absolute value of hyperpolarizabilities, ${ }^{8,10}$ and this error increases with the system size. However, with some specially developed exchange-correlation (XC) potentials such as the SAOP potential, ${ }^{16}$ the exact-exchange potential, ${ }^{17}$ as well as the hybrid potential with long range correction, ${ }^{18}$ TDDFT yields more accurate values for hyperpolarizabilities. Taking into account the efficiency of DFT, this makes TDDFT an appealing method in the calculation of dynamic hyperpolarizabilities. Up to now, all TDDFT calculations for the dynamic hyperpolarizabilities are performed in frequency domain.

Localized density matrix (LDM) method is an $\mathrm{O}(\mathrm{N})$ density matrix based method ${ }^{19-21}$ and was developed to calculate the excited state properties of very large systems. It has been implemented at TDDFT level, and the resulting TDDFT/ LDM method was used to calculate the absorption spectra and excited state energies. ${ }^{22-24}$ In the present work we generalize the TDDFT/LDM method to calculate dynamic hy-

\footnotetext{
${ }^{a)}$ Author to whom correspondence should be addressed. Electronic mail: ghc@everest.hku.hk
}

perpolarizabilities. Two approaches are adopted. We may integrate the density matrix based time-dependent Kohn-Sham (TDKS) equation in real time domain. This is a highly intuitive way to calculate the dynamic hyperpolarizabilities and is easy to implement. In addition, higher order nonlinear response can also be calculated in this way. Since the calculation of the derivative of XC potential with respect to the electron potential is avoided, it allows the use of complicated forms of XC potential such as orbital-dependent XC functions ${ }^{25}$ and optimized effective potentials. ${ }^{26-28}$ Alternatively we may start with the density matrix based timedependent Kohn-Sham equation and calculate dynamic hyperpolarizabilities in frequency domain following the works of Furche, ${ }^{29}$ Tretiak and Mukamel, ${ }^{30}$ and Tretiak and Chernyak. ${ }^{31}$ With this approach we show that it is straightforward to derive the $2 n+1$ rule $^{32}$ which is used to calculate the hyperpolarizabilites by avoiding the calculation of the density matrix of the same order. The paper is organized as follows. In Sec. II A we discuss the density matrix based TDDFT in real time domain and the approaches to integrate the equation of motion. The formulation for the second-order density matrix in frequency domain with density matrix based TDDFT as well as the $2 n+1$ rule to calculate the dynamic hyperpolarizabilities are discussed in Sec. II B. In Sec. III, calculations for the dynamic hyperpolarizabilities of some small molecules are reported for both approaches, and the results are presented.

\section{THEORY}

\section{A. Density matrix based TDDFT in real time domain}

For a system initially at its ground state and perturbed by a time-dependent external potential, the reduced singleelectron density matrix of this system within TDDFT satisfies the TDKS equation, ${ }^{6}$ 


$$
i \dot{P}(t)=[F(t), P(t)],
$$

with initial condition $P(t=0)=P_{0}$, where $P(t)$ and $F(t)$ are density matrix and Fock matrix, respectively. Various methods have been proposed to solve this equation of motion. Here we present only the most efficient methods according to our experience: the exponential midpoint method $(\mathrm{EMM}),{ }^{33,34}$ the Magnus method, ${ }^{34,35}$ and the Runge-Kutta method in the interaction representation. ${ }^{36,37}$

In EMM $P(t+\Delta t)$ is calculated with the following equation:

$$
P(t+\Delta t)=e^{-i F(t+\Delta t / 2) \Delta t} P(t) e^{i F(t+\Delta t / 2) \Delta t} .
$$

To calculate $F(t+\Delta t / 2)$, the density matrix at $t+\Delta t / 2$ is determined by

$$
P(t+\Delta t / 2)=e^{-i F(t) \Delta t / 2} P(t) e^{i F(t) \Delta t / 2} .
$$

This is different from that proposed in Ref. 33. According to our experience, this formulation is more stable. It can be seen from Eqs. (2) and (3) that the Fock matrix has to be constructed twice in each time step, and the construction of the Fock matrix is the most time-consuming part of computation. $e^{-i F \Delta t}$ can be calculated by diagonalizing the Fock matrix if its dimension is not too large. Other methods have been $\operatorname{proposed}^{34}$ to calculate $e^{-i F \Delta t}$ efficiently without diagonalizing $F$.

In the Magnus method $P(t+\Delta t)$ is calculated based on

$$
\begin{aligned}
P(t+\Delta t)= & e^{-i\left\{\left(F\left(t_{1}\right)+F\left(t_{2}\right) / 2+i(\sqrt{3} / 12) \Delta t\left[F\left(t_{1}\right), F\left(t_{2}\right)\right]\right\}\right.} \\
& \times P(t) e^{i\left\{\left(F\left(t_{1}\right)+F\left(t_{2}\right) / 2+i(\sqrt{3} / 12) \Delta t\left[F\left(t_{1}\right), F\left(t_{2}\right)\right]\right\}\right.},
\end{aligned}
$$

where $t_{1,2}=t+(1 / 2 \mp \sqrt{3} / 6) \Delta t . P\left(t_{1}\right)$ and $P\left(t_{2}\right)$, which are needed to calculate $F\left(t_{1}\right)$ and $F\left(t_{2}\right)$, are determined with the following equation:

$$
\begin{aligned}
& P\left(t_{1}\right)=e^{-i F(t)\left(t_{1}-t\right)} P(t) e^{i F(t)\left(t_{1}-t\right)}, \\
& P\left(t_{2}\right)=e^{-i F\left(t_{1}\right)\left(t_{2}-t_{1}\right)} P\left(t_{1}\right) e^{i F\left(t_{1}\right)\left(t_{2}-t_{1}\right)} .
\end{aligned}
$$

It can be seen from Eqs. (4) and (5) that the Fock matrix needs to be constructed three times in each time step.

To integrate Eq. (1) with the Runge-Kutta method, the time step is decided by the largest absolute eigenvalue of the Fock matrix. A much larger time step can be used by transforming Eq. (1) to the interaction representation

$$
i \dot{P}_{I}(t)=\left[F_{I}(t)-F_{0}, P_{I}(t)\right],
$$

where $P_{I}(t)=e^{-i F_{0} t} P(t) e^{-i F_{0} t}, F_{I}(t)=e^{-i F_{0} t} F(t) e^{-i F_{0} t} . F_{0}$ is usually chosen as the ground state Fock matrix. In addition, the fifth-order Runge-Kutta method with adaptive time steps by Cash and Karp can also be applied to solve Eq. (6). ${ }^{36}$ Unlike the EMM and the Magnus method, the idempotency condition of the density matrix is not assured automatically and usually a small time step is required. The Fock matrix needs to be built four times each step with the fourth-order RungeKutta method and six times in the fifth-order Runge-Kutta method. An advantage using the fourth-order Runge-Kutta method in the interaction representation is that the exponential of the Fock matrix only needs to be calculated once due to the even time step, while it has to be calculated at each step in EMM and the Magnus method. This makes the fourth-order Runge-Kutta method in the interaction representation a better candidate for large systems. However, if the external field is very strong, the largest absolute eigenvalue of $F_{I}(t)-F_{0}$ could still be very large and a small time step has to be used to assure stable simulation. In this case, higher efficiency may be achieved by choosing $F_{0}$ properly.

The efficiency and accuracy of these three methods are comparable because the most time-consuming step is the construction of the Fock matrix. These methods are all low order methods and very small time step has to be used to obtain highly accurate solution of Eq. (1). High order methods such as Chebyshev ${ }^{38}$ and Legendre ${ }^{39}$ methods can lead to much higher accuracy and are more efficient if high accuracy is required. However, if relatively low accuracy is desired, these high order methods are too expensive.

The time-dependent first-order and second-order density matrices can be obtained through a perturbation expansion of Eq. (1),

$$
\begin{aligned}
& i \dot{P}^{(1)}=\left[F_{0}, P^{(1)}\right]+\left[h^{(1)}, P_{0}\right], \\
& i \dot{P}^{(2)}=\left[F_{0}, P^{(2)}\right]+\left[h^{(2)}, P_{0}\right]+\left[h^{(1)}, P^{(1)}\right],
\end{aligned}
$$

where $h^{(1)}$ and $P^{(1)}$ are the first-order Fock matrix and density matrix, respectively, and $h^{(2)}$ and $P^{(2)}$ are the secondorder Fock matrix and density matrix, respectively. The solution for Eq. (7) has been discussed in our previous work $^{22-24}$ and the Chebyshev expansion method ${ }^{24,40,41}$ is shown to be highly efficient and accurate to solve Eq. (7) when the external perturbation is a $\delta$ function in time. In addition, dynamic polarizabilities at a large energy range can be determined at the same time through the solution of Eq. (7). On the other hand, it is more expensive to evaluate hyperpolarizabilities via Eqs. (7) and (8) than via Eq. (1).

The dynamic hyperpolarizabilities can be calculated via Eq. (1) in the following way. When an electric field with a certain frequency is turned on at $t=0$, the corresponding time-dependent density matrix can be obtained by integrating Eq. (1), and the time-dependent dipole moment is thus determined. The dipole moment component with frequency that is twice the input frequency corresponds to the first dynamic hyperpolarizabilities. The dynamic hyperpolarizabilities due to two different frequencies such as two wave mixing can also be determined in a similar way. It should be noted that the strength of the external field can be neither too weak to discern the second-order response nor too strong to induce higher order response. In addition, when the electric field is turned on suddenly, nonadiabatic response could arise. To avoid this, the electric field has to be turned on slowly. Furthermore, it is not straightforward to extract the values of individual spatial components of higher order hyperpolarizabilities with this time-domain method. To extract the second-order response embedded in the time-dependent dipole moment, we adopt the filter diagonalization method, ${ }^{42-44}$ which has been shown to be highly accurate in spectral analysis. 


\section{B. Density matrix based quadratic response TDDFT in frequency domain}

Following Ref. 29, the first-order and second-order changes of the density matrix due to a perturbation with a certain frequency satisfy

$$
\begin{aligned}
& P_{0} P^{(a)}+P^{(a)} P_{0}=P^{(a)}, \\
& \omega_{a} P^{(a)}=\left[F_{0}, P^{(a)}\right]+\left[h^{(a)}, P_{0}\right], \\
& P_{0} P^{(a b)}+P^{(a b)} P_{0}+P^{(a)} P^{(b)}+P^{(b)} P^{(a)}=P^{(a b)}, \\
&\left(\omega_{a}+\omega_{b}\right) P^{(a b)}= {\left[F_{0}, P^{(a b)}\right]+\left[h_{a b}^{(2)}, P_{0}\right]+\left[h_{a}^{(1)}, P^{(b)}\right] } \\
&+ {\left[h_{b}^{(1)}, P^{(a)}\right], }
\end{aligned}
$$

where $\omega_{a}\left(\omega_{b}\right)$ is the frequency of external perturbation $a(b)$, $P_{0}$ and $F_{0}$ are the ground state density matrix and Fock matrix, respectively, $P^{(a)}$ and $h^{(a)}$ are the first-order change of the density matrix and Fock matrix due to external perturbation $a$, respectively, and $P^{(a b)}$ and $h^{(a b)}$ are the second-order change of the density matrix and Fock matrix due to external perturbations $a$ and $b$, respectively. Equations (9) and (11) are idempotency conditions for the first- and second-order density matrices, respectively. The first-order Fock matrix $h^{(a)}$ depends on $P^{(a)}$ and satisfies

$$
h^{(a)}=F^{(1)}\left\{P^{(a)}\right\}+f^{a},
$$

where $f^{a}$ is the matrix of the external perturbation $a$, and $F^{(1)}\{P\}$ is the first-order change of the Fock matrix with respect to density matrix change $P$ and is calculated as follows:

$$
\begin{aligned}
\left(F^{(1)}\{P\}\right)_{p q}= & \iint \varphi_{p}\left(\mathbf{r}_{1}\right) \varphi_{q}\left(\mathbf{r}_{1}\right)\left\{\frac{1}{\left|\mathbf{r}_{1}-\mathbf{r}_{2}\right|}\right. \\
& \left.+\left.\frac{\partial V_{x c}\left(\mathbf{r}_{1}\right)}{\partial \rho\left(\mathbf{r}_{2}\right)}\right|_{\rho=\rho_{0}}\right\} \rho\left(\mathbf{r}_{2}\right) d r_{1} d r_{2},
\end{aligned}
$$

where $p, q$ are indices for basis functions, $\varphi_{p}$ and $\varphi_{q}$ are basis functions, $V_{\mathrm{XC}}$ is the $\mathrm{XC}$ potential, $\rho_{0}$ is the ground state density, and $\rho\left(\mathbf{r}_{2}\right)$ is the electron density corresponding to density matrix $P$. Note that the adiabatic approximation ${ }^{6}$ for the XC potential has been applied in Eq. (14). The secondorder Fock matrix $h^{(a b)}$ can be determined by

$$
h^{(a b)}=F^{(1)}\left\{P^{(a b)}\right\}+F^{(2)}\left\{P^{(a)}, P^{(b)}\right\},
$$

where $F^{(2)}\left\{P_{1}, P_{2}\right\}$ is the second-order change of the Fock matrix with respect to two density matrices $P_{1}$ and $P_{2}$ and it reads

$$
\begin{aligned}
& \left.{ }^{\left(F^{(2)}\right.}\left\{P_{1}, P_{2}\right\}\right)_{p q} \\
& =\iiint \varphi_{p}\left(\mathbf{r}_{1}\right) \varphi_{q}\left(\mathbf{r}_{1}\right) \\
& \times\left.\frac{\partial^{2} V_{x c}\left(\mathbf{r}_{1}\right)}{\partial \rho\left(\mathbf{r}_{2}\right) \partial \rho\left(\mathbf{r}_{3}\right)}\right|_{\rho=\rho_{0}} \rho_{1}\left(\mathbf{r}_{2}\right) \rho_{2}\left(\mathbf{r}_{3}\right) d r_{1} d r_{2} d r_{3},
\end{aligned}
$$

where $\rho_{1}$ and $\rho_{2}$ are electron densities corresponding to density matrices $P_{1}$ and $P_{2}$, respectively. If $P_{1}$ and $P_{2}$ are the same, there is a prefactor of $1 / 2$ in Eq. (16). The above equations are formulated in any orthogonormalized basis set and we only deal with the canonical molecular orbital basis in the following. $P_{0}$ and $F_{0}$ are diagonal in canonical molecular orbital basis. The occupied-occupied and virtualvirtual parts of $P^{(a)}$ are zero according to Eq. (9) and the virtual-occupied and occupied-virtual part of $P^{(a)}$ is decided by Eq. (10). Using Eq. (13), Eq. (10) can be further written as

$$
\omega_{a} P^{(a)}-\left[F_{0}, P^{(a)}\right]-\left[F^{(1)}\left\{P^{(a)}\right\}, P_{0}\right]=\left[f^{a}, P_{0}\right] .
$$

The linear response equation used to determine the firstorder change of density matrix in TDDFT can thus be expressed as

$$
\begin{gathered}
\left(\begin{array}{cc}
A & B \\
B & A
\end{array}\right)\left(\begin{array}{c}
P_{V O}^{(a)} \\
\left(P_{O V}^{(a)}\right)^{+}
\end{array}\right)-\omega_{a}\left(\begin{array}{cc}
1 & 0 \\
0 & -1
\end{array}\right)\left(\begin{array}{c}
P_{V O}^{(a)} \\
\left(P_{O V}^{(a)}\right)^{+}
\end{array}\right) \\
=-\left(\begin{array}{c}
f_{V O}^{a} \\
\left(f_{O V}^{a}\right)^{+}
\end{array}\right) .
\end{gathered}
$$

The matrices $A$ and $B$ are

$$
\begin{aligned}
A_{a i, b j}= & \Delta_{a i, b j}+B_{a i, b j}, \quad \Delta_{a i, b j}=\left(\varepsilon_{a}-\varepsilon_{i}\right) \delta_{a b} \delta_{i j}, \\
B_{a i, b j}= & \iint \varphi_{a}\left(\mathbf{r}_{1}\right) \varphi_{i}\left(\mathbf{r}_{1}\right)\left\{\frac{1}{\left|\mathbf{r}_{1}-\mathbf{r}_{2}\right|}\right. \\
& \left.+\left.\frac{\partial V_{x c}\left(\mathbf{r}_{1}\right)}{\partial \rho\left(\mathbf{r}_{2}\right)}\right|_{\rho=\rho_{0}}\right\} \varphi_{b}\left(\mathbf{r}_{2}\right) \varphi_{j}\left(\mathbf{r}_{2}\right) d r_{1} d r_{2},
\end{aligned}
$$

where nonhybrid XC functionals are adopted. The excitation energies can be determined by the poles of the first-order density matrix as ${ }^{45,46}$

$$
\left(\begin{array}{ll}
A & B \\
B & A
\end{array}\right)\left(\begin{array}{l}
X_{n} \\
Y_{n}
\end{array}\right)=\omega_{n}\left(\begin{array}{cc}
1 & 0 \\
0 & -1
\end{array}\right)\left(\begin{array}{l}
X_{n} \\
Y_{n}
\end{array}\right),
$$

where $X_{n}$ and $\left(Y_{n}\right)^{+}$are the virtual-occupied and occupiedvirtual part of the transition density matrix for the excited state $n$. Equation (18) is a set of linear equation and one can use either the sum-over-state method by solving all the excitation energies and transition density matrices in Eq. (21) or an iteration method to solve it. In the sum-over-state expression, $P_{V O}^{(a)}$ and $P_{O V}^{(a)}$ satisfy ${ }^{29}$

$$
\left(\begin{array}{c}
P_{V O}^{(a)} \\
\left(P_{O V}^{(a)}\right)^{+}
\end{array}\right)=\sum_{n} \frac{\operatorname{Tr}\left(f_{O V}^{a}\left(X_{n}+Y_{n}\right)\right)}{\omega_{a}-\omega_{n}}\left(\begin{array}{c}
X_{n} \\
Y_{n}
\end{array}\right),
$$

where the fact that $f^{a}$ is Hermitian has been used. In the iteration method, the following iteration scheme is proved to be very efficient: ${ }^{47,48}$

$$
\left(P_{V O}^{(a)}+\left(P_{O V}^{(a)}\right)^{+}\right)=-\frac{\Delta}{\Delta^{2}-\omega_{a}^{2}}\left[2 f_{V O}^{a}+2 B\left(P_{V O}^{(a)}+\left(P_{O V}^{(a)}\right)^{+}\right)\right],
$$

where $P_{V O}^{(a)}$ and $P_{O V}^{(a)}$ can be decided together with the following relation:

$$
P_{V O}^{(a)}-\left(P_{O V}^{(a)}\right)^{+}=\omega_{a} \Delta^{-1}\left(P_{V O}^{(a)}+\left(P_{O V}^{(a)}\right)^{+}\right) .
$$

When the first-order changes of density matrices $P^{(a)}$ and $P^{(b)}$ are decided, the second-order change of density matrix $P^{(a b)}$ can be determined by Eqs. (11) and (12). The occupied- 
occupied and virtual-virtual part of $P^{(a b)}$ can be decided directly from the idempotency condition in Eq. (11),

$$
\begin{aligned}
& \left(P^{(a b)}\right)_{O O}=-\left(P^{(a)} P^{(b)}+P^{(b)} P^{(a)}\right)_{O O}, \\
& \left(P^{(a b)}\right)_{V V}=\left(P^{(a)} P^{(b)}+P^{(b)} P^{(a)}\right)_{V V} .
\end{aligned}
$$

The virtual-occupied and occupied-virtual part of $P^{(a b)}$ can be decided by Eq. (12). Using the fact that $F^{(1)}\{P\}$ depends linearly on $\mathrm{P}$, we have the following equation:

$$
\begin{aligned}
\left(\omega_{a}\right. & \left.+\omega_{b}\right) P_{O V}^{(a b)}-\left[F_{0}, P_{O V}^{(a b)}\right]_{O V}-\left[F^{(1)}\left\{P_{O V}^{(a b)}+P_{V O}^{(a b)}\right\}, P_{0}\right]_{O V} \\
= & \left\{\left[h_{a}, P^{(b)}\right]+\left[h_{b}, P^{(a)}\right]+\left[F^{(2)}\left\{P^{(a)}, P^{(b)}\right\}, P_{0}\right]\right. \\
& \left.+\left[F^{(1)}\left\{P_{O O}^{(a b)}+P_{V V}^{(a b)}\right\}, P_{0}\right]\right\}_{O V},
\end{aligned}
$$

for the occupied-virtual part of $P^{(a b)}$, and the virtualoccupied part satisfies a similar equation. One can see that Eq. (26) has exactly the same structure as Eq. (17) for $P_{V O, O V}^{(a b)}$, and it can be written in the form of Eq. (18) as

$$
\begin{aligned}
& \left(\begin{array}{ll}
A & B \\
B & A
\end{array}\right)\left(\begin{array}{c}
P_{V O}^{(a b)} \\
\left(P_{O V}^{(a b)}\right)^{+}
\end{array}\right)-\left(\omega_{a}+\omega_{b}\right)\left(\begin{array}{cc}
1 & 0 \\
0 & -1
\end{array}\right)\left(\begin{array}{c}
P_{V O}^{(a b)} \\
\left(P_{O V}^{(a b)}\right)^{+}
\end{array}\right) \\
& =\left(\begin{array}{c}
-Q_{V O} \\
\left(Q_{O V}\right)^{+}
\end{array}\right)
\end{aligned}
$$

where $Q_{V O}$ and $Q_{O V}$ are virtual-occupied and occupiedvirtual parts of the right hand side of Eq. (26). Similar to the linear response equation, $P_{O V}^{(a b)}$ or $P_{V O}^{(a b)}$ can be decided either through the sum-over-state method or through the iteration method. In the sum-over-state method, we have

$$
\left(\begin{array}{c}
P_{V O}^{(a b)} \\
\left(P_{O V}^{(a b)}\right)^{+}
\end{array}\right)=\sum_{n} \frac{\left.\operatorname{Tr}\left(Q_{V O}^{+} X_{n}-Q_{O V} Y_{n}\right)\right)}{\left(\omega_{a}+\omega_{b}\right)-\omega_{n}}\left(\begin{array}{c}
X_{n} \\
Y_{n}
\end{array}\right)
$$

Within the iteration method, we have the following scheme:

$$
\begin{aligned}
\left(P_{V O}^{a b}+\left(P_{O V}^{a b}\right)^{+}\right)= & -\frac{\Delta}{\Delta^{2}-\left(\omega_{a}+\omega_{b}\right)^{2}}\left[\left(Q_{V O}-Q_{O V}^{+}\right)\right. \\
& +2 B\left(X^{(a)}+Y^{(a)}\right)+\frac{\left(\omega_{a}+\omega_{b}\right)}{\Delta} \\
& \left.\times\left(Q_{V O}+Q_{O V}^{+}\right)\right]
\end{aligned}
$$

where $P_{V O}^{(a b)}$ and $P_{O V}^{(a b)}$ can be decided together with

$$
\begin{aligned}
\left(P_{V O}^{a b}-\left(P_{O V}^{a b}\right)^{+}\right)= & \frac{\omega_{a}+\omega_{b}}{\Delta}\left(P_{V O}^{a b}+\left(P_{O V}^{a b}\right)^{+}\right) \\
& -\frac{1}{\Delta}\left(Q_{V O}+Q_{O V}^{+}\right) .
\end{aligned}
$$

The dynamic polarizabilities and hyperpolarizabilities can be calculated when the first-order and second-order density matrices due to external electric field are determined. The dynamic polarizability $\alpha_{b a}\left(-\omega_{a} ; \omega_{a}\right)$ can be calculated through the first-order density matrix,

$$
\alpha_{b a}\left(-\omega_{a} ; \omega_{a}\right)=\operatorname{Tr}\left(\mu^{b} P^{(a)}\right)=\operatorname{Tr}\left[\mu_{O V}^{b}\left(P_{V O}^{(a)}+\left(P_{O V}^{a}\right)^{+}\right)\right],
$$

where $\mu^{b}$ is the dipole moment matrix along the $b$ direction, and $P^{(a)}$ is the first-order density matrix due to an electric field along the $a$ direction with frequency $\omega_{a}$. Similarly, the dynamic hyperpolarizabilities can be calculated through the second-order density matrix,

$$
\begin{aligned}
\beta_{c a b}( & \left.\left(\omega_{a}+\omega_{b}\right) ; \omega_{a}, \omega_{b}\right) \\
= & \operatorname{Tr}\left(\mu^{c} P^{(a b)}\right) \\
= & \operatorname{Tr}\left[\mu_{O V}^{c}\left(P_{V O}^{(a b)}+\left(P_{O V}^{a b}\right)^{+}\right)\right]+\operatorname{Tr}\left(\mu_{O O}^{c} P_{O O}^{(a b)}\right) \\
& \times+\operatorname{Tr}\left(\mu_{V V}^{c} P_{V V}^{(a b)}\right) .
\end{aligned}
$$

Using Eq. (28), we have the following equation for the first term on the right hand side of Eq. (32):

$$
\begin{aligned}
\operatorname{Tr} & {\left[\mu_{O V}^{c}\left(P_{V O}^{(a b)}+\left(P_{O V}^{a b}\right)^{+}\right)\right] } \\
& =\sum_{n} \frac{\operatorname{Tr}\left(Q_{V O}^{+} X_{n}-Q_{O V} Y_{n}\right) \operatorname{Tr}\left(\mu_{O V}^{c} X_{n}+\mu_{V O}^{c} Y_{n}\right)}{\left(\omega_{a}+\omega_{b}\right)-\omega_{n}} \\
& =\operatorname{Tr}\left[Q_{V O}^{+} P_{V O}^{(c)}-Q_{O V}\left(P_{O V}^{(c)}\right)^{+}\right],
\end{aligned}
$$

where the sum-over-state expression for $P^{(c)}$ due to an external field along the $c$ direction with frequency $\omega_{a}+\omega_{b}$ has been used. It can be seen that with Eq. (33), the second-order change of density matrix is actually not needed to calculate dynamic hyperpolarizabilities. This is the dynamic hyperpolarizability expression with the $2 n+1$ rule. Using Eqs. (25), (26), (32), and (33), we can reach the following expression for the dynamic hyperpolarizability based on the $2 n+1$ rule:

$$
\begin{aligned}
& \beta_{a b c}\left(\omega_{a} ; \omega_{b}, \omega_{c}\right)= \operatorname{Tr}\left\{\begin{array}{l}
\sum_{\text {permutation }} P_{O V}^{(a)} h_{V V}^{b} P_{V O}^{(c)} \\
\text { over } a, b, c
\end{array}\right\} \\
&-\operatorname{Tr}\left\{\begin{array}{l}
\sum_{\begin{array}{l}
\text { permutation } \\
\text { over } a, b, c
\end{array}} P_{V O}^{(a)} h_{O O}^{b} P_{O V}^{(c)} \\
\end{array}\right\} \\
&+g_{\mathrm{XC}}\left(\rho^{(a)}, \rho^{(b)}, \rho^{(c)}\right),
\end{aligned}
$$

where $g_{\mathrm{XC}}$ satisfy

$$
\begin{aligned}
g_{\mathrm{XC}}\left(\rho^{(a)}, \rho^{(b)}, \rho^{(c)}\right)= & \iiint \rho^{(a)}\left(\mathbf{r}_{1}\right) \rho^{(b)}\left(\mathbf{r}_{2}\right) \rho^{(c)}\left(\mathbf{r}_{3}\right) \\
& \times \frac{\delta^{3} E_{\mathrm{XC}}}{\delta \rho\left(\mathbf{r}_{1}\right) \delta \rho\left(\mathbf{r}_{2}\right) \delta \rho\left(\mathbf{r}_{3}\right)} d r_{1} d r_{2} d r_{3},
\end{aligned}
$$

where $\rho^{(a)}, \rho^{(b)}$, and $\rho^{(c)}$ are the first-order change of electron density, and $\omega_{a}, \omega_{b}$, and $\omega_{a}$ satisfy $\omega_{a}+\omega_{b}+\omega_{c}=0$. To obtain Eq. (34), the following relations have been used:

$$
\begin{aligned}
& P^{(a)}\left(\omega_{a}\right)=\left(P^{(a)}\left(-\omega_{a}\right)\right)^{+}, \\
& F^{(1)}\left(P^{(a)}\left(\omega_{a}\right)\right)=F^{(1)}\left(P^{(a)}\left(-\omega_{a}\right)\right), \\
& \operatorname{Tr}\left[\left(P_{V O}^{(a)}\right)^{+} F_{V O}^{(1)}\left\{P_{O O}^{(b c)}\right\}+\left(P_{O V}^{(a)}\right)^{+} F_{O V}^{(1)}\left\{P_{O O}^{(b c)}\right\}\right] \\
& \quad=\operatorname{Tr}\left[P_{O O}^{(b c)} F_{O O}^{(1)}\left\{P^{(a)}\right\}\right],
\end{aligned}
$$




$$
\begin{aligned}
& \operatorname{Tr}\left[\left(P_{V O}^{(a)}\right)^{+} F_{V O}^{(1)}\left\{P_{V V}^{(b c)}\right\}+\left(P_{O V}^{(a)}\right)^{+} F_{O V}^{(1)}\left\{P_{V V}^{(b c)}\right\}\right] \\
& \quad=\operatorname{Tr}\left[P_{V V}^{(b c)} F_{V V}^{(1)}\left\{P^{(a)}\right\}\right] .
\end{aligned}
$$

Using Eqs. (22) and (34), a sum-over-state expression for the dynamic hyperpolarizabilities similar to that in Ref. 31 can be also reached.

It can be seen that the above derivation is much simple than proposed before. ${ }^{7,32}$ Similar strategy can be used to obtain the $2 n+1$ expression for third-order response properties. It is worth noting that the second-order change of the density matrix itself cannot be obtained through the $2 n+1$ rule, instead, only the second-order response properties can be achieved through the $2 n+1$ rule. The two-photon absorption (TPA) cross section can be determined by the poles of dynamic hyperpolarizability ${ }^{49,50}$ as

$$
\lim _{2 \omega \rightarrow \omega_{n}}\left(2 \omega-\omega_{n}\right) \beta_{a b c}(-2 \omega ; \omega, \omega)=-\sigma_{b c}\left(\omega_{n}\right)\left\langle n\left|\mu^{a}\right| 0\right\rangle,
$$

and the TPA cross section can be obtained based on Eq. (34) as the following relation:

$$
\begin{aligned}
\sigma_{a b}\left(\omega_{n}\right)= & -\operatorname{Tr}\left[P_{O V}^{(a)}\left(h_{V V}^{b} Y_{n}+h_{V V}^{n} P_{V O}^{(b)}\right)+P_{O V}^{(b)}\left(h_{V V}^{a} Y_{n}\right.\right. \\
& \left.\left.+h_{V V}^{n} P_{V O}^{(a)}\right)+X_{n}^{+}\left(h_{V V}^{a} P_{V O}^{(b)}+h_{V V}^{b} P_{V O}^{(a)}\right)\right]+\operatorname{Tr}\left[P_{V O}^{(a)}\right. \\
& \times\left(h_{O O}^{b} X_{n}^{+}+h_{O O}^{n} P_{O V}^{(b)}\right)+P_{V O}^{(b)}\left(h_{O O}^{a} X_{n}^{+}+h_{O O}^{n} P_{O V}^{(a)}\right) \\
& \left.+Y_{n}\left(h_{O O}^{a} P_{O V}^{(b)}+h_{O O}^{b} P_{O V}^{(a)}\right)\right]-g_{X C}\left(\rho^{(a)}, \rho^{(b)}, \rho^{n}\right),
\end{aligned}
$$

where the frequency for $a$ and $b$ is $\omega_{n} / 2, h^{n}=F^{(1)}\left\{P^{(n)}\right\}$, and $P^{(n)}$ and $\rho^{n}$ are the transition density matrix and transition density for the state $n$. The dipole transition between two excited states $m$ and $n$ can also be decided by the poles of the dynamic hyperpolarizability:

$$
\begin{aligned}
& \left.\lim _{\omega_{b} \rightarrow-\omega_{m} \omega_{c} \rightarrow \omega_{n}} \lim _{b}+\omega_{m}\right)\left(\omega_{c}-\omega_{n}\right) \beta_{a b c}\left(-\left(\omega_{a}+\omega_{b}\right) ; \omega_{b}, \omega_{c}\right) \\
& \quad=-\left\langle m\left|\mu^{a}-d_{a}\right| n\right\rangle\left\langle n\left|\mu^{c}\right| 0\right\rangle\left\langle 0\left|\mu^{b}\right| m\right\rangle .
\end{aligned}
$$

The transition dipole between two excited states thus reads

$$
\begin{aligned}
\left\langle m\left|\mu^{a}-d_{a}\right| n\right\rangle= & -\operatorname{Tr}\left[P_{O V}^{(a)}\left(h_{V V}^{m} X_{n}+h_{V V}^{n} Y_{m}\right)+Y_{n}^{+}\left(h_{V V}^{a} Y_{m}\right.\right. \\
& \left.\left.+h_{V V}^{m} P_{V O}^{(a)}\right)+X_{m}^{+}\left(h_{V V}^{a} X_{n}+h_{V V}^{n} P_{V O}^{(a)}\right)\right] \\
& +\operatorname{Tr}\left[P_{V O}^{(a)}\left(h_{O O}^{m} Y_{n}+h_{O O}^{n} X_{m}\right)+X_{n}\left(h_{O O}^{a} X_{m}^{+}\right.\right. \\
& \left.\left.+h_{O O}^{m} P_{O V}^{(a)}\right)+Y_{m}\left(h_{O O}^{a} Y_{n}^{+}+h_{V V}^{n} P_{V O}^{(a)}\right)\right] \\
& -g_{\mathrm{XC}}\left(\rho^{(a)}, \rho^{m}, \rho^{n}\right),
\end{aligned}
$$

where the frequency for $a$ is $\omega_{m}-\omega_{n}$, and $d_{a}$ is the ground state dipole moment of the $a$ direction. In fact, the matrix element of any one-electron operator between two different excited states can also be calculated with the second-order density matrix using Eqs. (25) and (26) with the following ingredients:

$$
\begin{aligned}
& P_{V O}^{(a)}=Y_{m}, \quad P_{O V}^{(a)}=X_{m}^{+}, \quad P_{V O}^{(b)}=X_{n}, \quad P_{O V}^{(b)}=Y_{n}^{+}, \\
& h_{a}=F^{(1)}\left(P^{(a)}\right), \quad h_{b}=F^{(1)}\left(P^{(b)}\right),
\end{aligned}
$$

$$
\langle m|\hat{A}| n\rangle=\operatorname{Tr}\left[P^{(a b)} A\right] .
$$

A particularly interesting case is the nonadiabatic (NA) coupling terms, where $\hat{A}$ is the derivative of Hamiltonian with respect to nuclei coordinate. The NA terms between excited state and the ground state have been proposed in Refs. 37 and 51. Here the NA terms among excited states can also be decided using the above formula. When the basis functions do not depend on the nuclei coordinates, Eq. (44) can be used directly to decide the NA terms. On the other hand, additional terms that are related to the derivatives of basis functions with respect to nuclear coordinates arise. When $m$ and $n$ are the same state, this second-order density matrix is just the difference between the excited state density matrix and the ground state density matrix, and excited state properties such as excited state dipole moment can thus be calculated from this excited state density matrix. ${ }^{52}$

\section{COMPUTATIONAL DETAILS AND RESULTS}

The solution of Eq. (1) using EMM, Magnus, fourthorder Runge-Kutta, or Runge-Kutta method with adaptive time steps in the interaction representation as well as the calculation of dynamic polarizabilities and hyperpolarizabilities using the above mentioned approaches are implemented in LODESTAR program package. ${ }^{53}$ In the following calculations, fourth-order Runge-Kutta method is used to solve Eq. (1) unless mentioned explicitly. LDA and adiabatic local density approximation are used for ground state and timedependent exchange-correlation potentials.

\section{A. Time-dependent dipole moment and dynamic polarizabilities for $\mathrm{C}_{2} \mathrm{H}_{4}$}

We calculate the time-dependent dipole moment along the $\mathrm{C}-\mathrm{C}$ direction for $\mathrm{C}_{2} \mathrm{H}_{4}$ due to an external electric field along the same direction, and the external field is a $\delta$ function in the time domain. When the external electric field is very weak, the time-dependent dipole moment should agree very well with the linear response results based on Eq. (7), which is determined using the Chebyshev method with high accuracy. 6-13G basis set and a time step of $0.01 \mathrm{fs}$ are adopted in the calculation. The time-dependent dipole moments due to the external fields of 0.05 and $0.5 \mathrm{eV} / \mathrm{bohr}$ by Eqs. (1) and (7) are plotted in Fig. 1. It can be seen that the time-dependent dipole moment calculated by Eq. (1) agrees very well with that calculated by Eq. (7) for the weak field case. For the strong field case, significant difference arises due to high order effects, as shown in Fig. 1. With Eq. (1) or (7), it is possible to calculate the dynamic polarizabilities over large energy range by taking Fourier transformation of the induced dipole moment. To obtain the dynamic polarizabilities from the time-dependent dipole moment, a total propagation time of $70 \mathrm{fs}$ is applied for solving Eq. (7) and a dephasing of $0.1 \mathrm{eV}$ is adopted in the Fourier transformation. This dephasing corresponds to an imaginary part of $0.1 \mathrm{eV}$ in the frequency. In addition, the error of the dynamic polarizabilities according to the time-domain method is of order $e^{-\Gamma T}$, where $\Gamma$ is the dephasing factor and $T$ is the total propagation time. The calculated real and imaginary parts of 

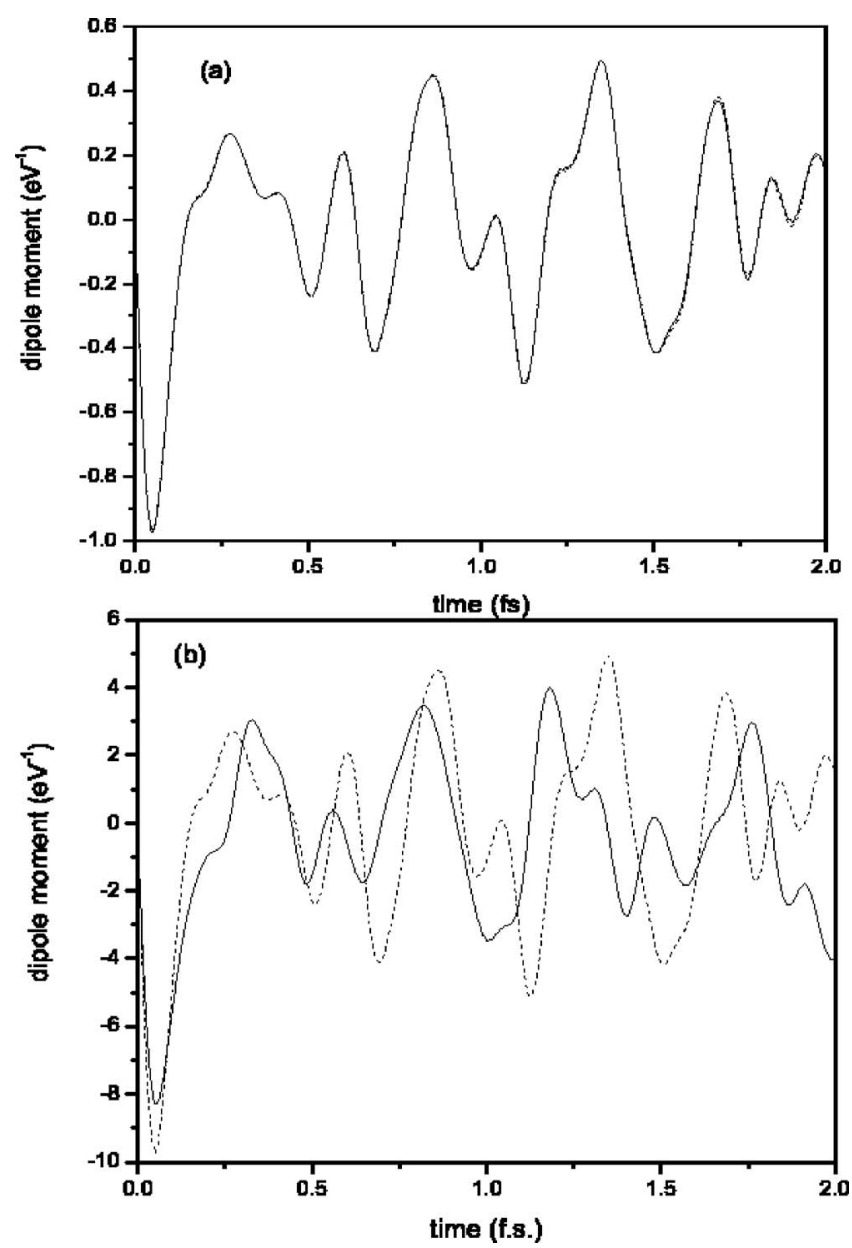

FIG. 1. The time-dependent dipole moment of $\mathrm{C}_{2} \mathrm{H}_{4}$ due to external electric field (dashed line: results due to time-domain TDDFT; solid line: results based on linear response time-domain TDDFT). (a) Field strength of $0.05 \mathrm{eV} / \mathrm{bohr}$ and (b) field strength $0.5 \mathrm{eV} / \mathrm{bohr}$.

the dynamic polarizabilities through time-domain and frequency domain TDDFTs are plotted in Fig. 2. It can be seen from this figure that dynamic polarizabilities from timedomain and frequency-domain TDDFTs agrees very well with each other. Time-domain TDDFT is more efficient in the calculation of dynamic polarizabilities than the frequency-domain TDDFT. This is because in the frequency domain, the dynamic polarizabilities have to be calculated one frequency at each time, while all the frequencies in an energy range can be calculated at the same time within the time-domain TDDFT.

\section{B. Dynamic hyperpolarizabilities}

To calculate dynamic hyperpolarizabilities using timedomain TDDFT, we have to calculate them one frequency at a time. In the calculations, a time-dependent electric field is applied as in Ref. 54 in the following form:

$$
E(t)=\left\{\begin{array}{cc}
(E \mathbf{r} \omega t \sin \omega t) /(2 n \pi), & 0 \leqslant t \leqslant 2 n \pi / \omega \\
E \mathbf{r} \sin \omega t, & t \geqslant 2 n \pi / \omega .
\end{array}\right.
$$

From Eq. (45), the electric field is fully turned on at the end of the $n$th cycle. This is to reduce the nonadiabatic effect. In the calculations for dynamic hyperpolarizabilities, $n$ is set to
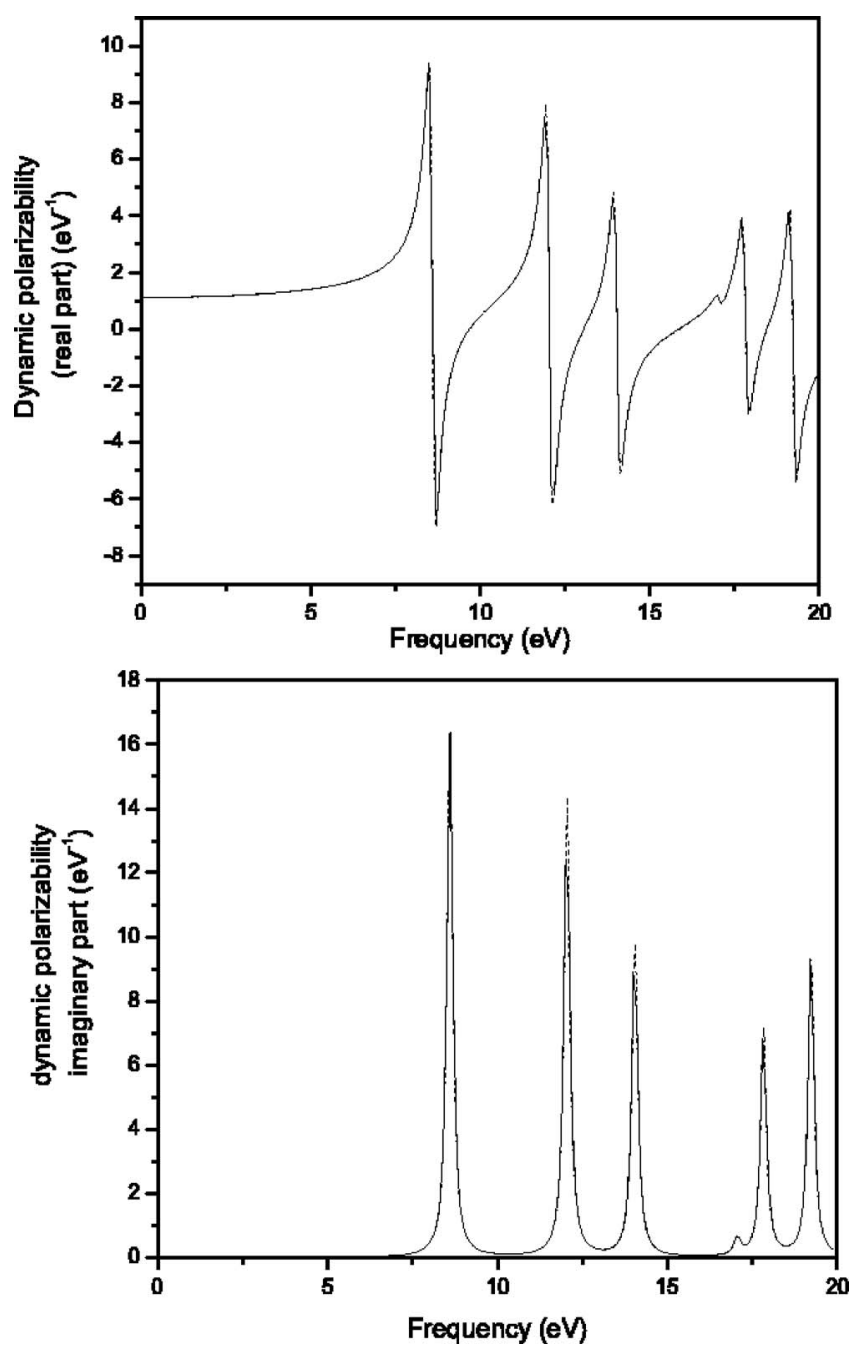

FIG. 2. The real and imaginary parts of the dynamic polarizabilities of $\mathrm{C}_{2} \mathrm{H}_{4}$ (dashed line: results due to time-domain TDDFT; solid line: results based on frequency-domain TDDFT).

1. An electric field of $0.1 \mathrm{eV} / \mathrm{bohr}$ is applied to calculate the dynamic hyperpolarizabilities. The time-dependent dipole moment of $\mathrm{C}_{2} \mathrm{H}_{4}$ along the $\mathrm{C}-\mathrm{C}$ direction due to this electric field described by Eq. (45) and the electric field with a sudden switch on is plotted in Fig. 3 together with the external field. It can be seen clearly from this figure that significant nonadiabatic effects arise if the electric field is turned on abruptly due to the excitation of the electron to excited states. On the other hand, the time-dependent dipole moment follows closely with the external field if the external field is turned on slowly as in Eq. (45). The external field contains both $\omega$ and $-\omega$ frequency components and the hyperpolarizabilities by definition should correspond to the strength of the components with frequency $2 \omega$ and zero embedded in the time-dependent dipole moment. The strength of the $2 \omega$ is related to the SHG and the strength of zero frequency is related to the OR parameters. Another dynamic second hyperpolarizability, the EOPE, is actually the same as the OR parameters according to Eq. (34). It can be seen from the above analysis that the dynamic hyperpolarizabilities $\beta_{a b b}$ can be calculated through time-domain TDDFT by applying an electric field along the $b$ direction. To calculate $\beta_{a b c}$, we 

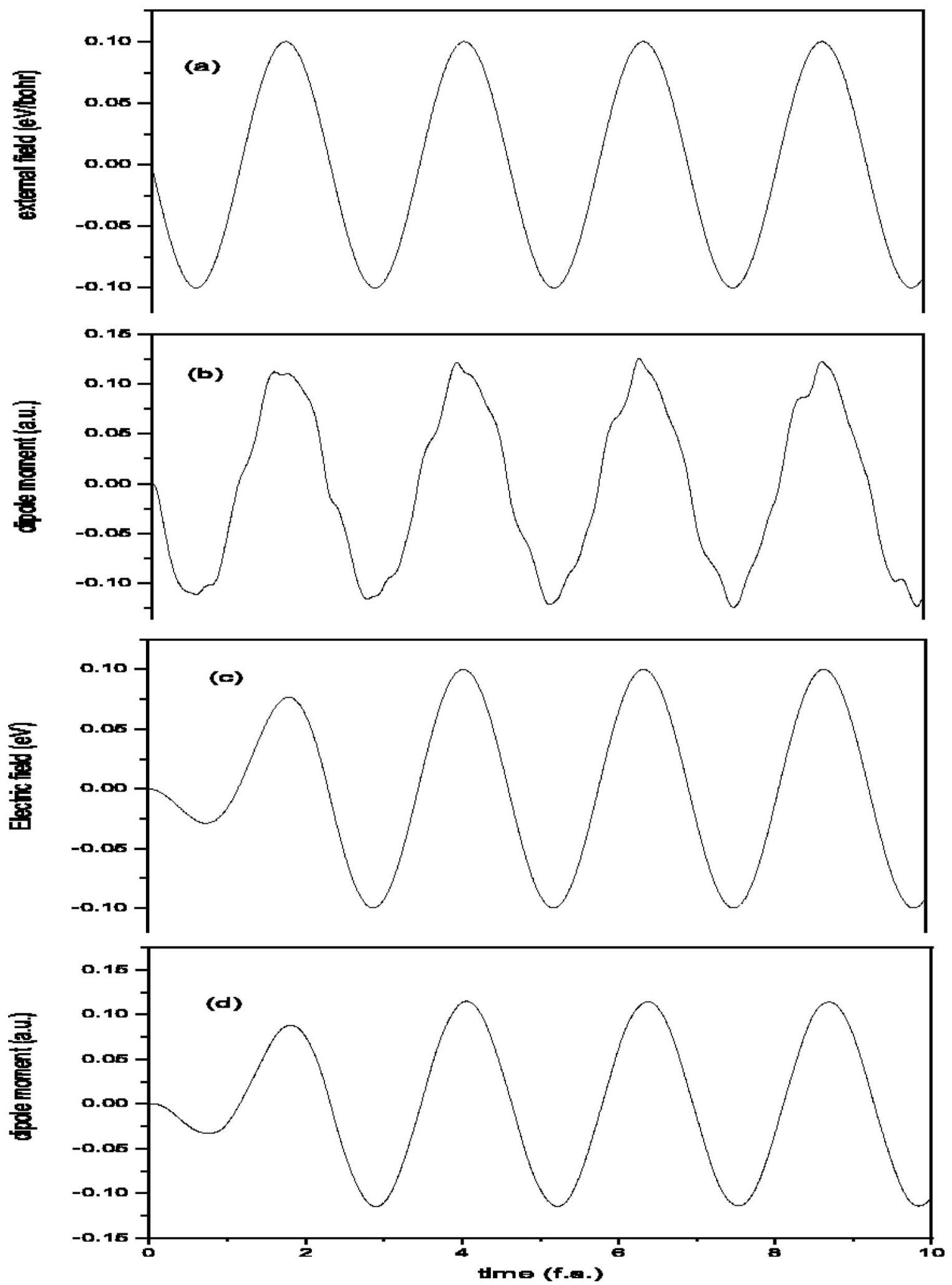

FIG. 3. The time-dependent dipole moment of $\mathrm{C}_{2} \mathrm{H}_{4}$ as well as the external electric field. (a) and (c) are the external electric fields, and (b) and (d) are corresponding time-dependent dipole moments.

need to apply two electric fields, one along the $b$ direction and the other along the $c$ direction. The strength of the component with frequency $2 \omega$ in the time-dependent dipole moment along the $a$ direction is thus the summation of $\beta_{a b b}$, $\beta_{a c c}$, and $\beta_{a b c}$, and $\beta_{a b c}$ can thus be determined. In the following we calculate the SHG and $\mathrm{OR}$ of $\mathrm{CO}, \mathrm{HF}, \mathrm{HCl}$, and
$\mathrm{LiF}$ with time-domain TDDFT and frequency-domain TDDFT based on Eq. (34). In the calculations, d-aug-ccpVTZ basis $^{55,56}$ is used. To achieve high accuracy for dynamic hyperpolarizabilities in time-domain TDDFT, a time-step of $0.001 \mathrm{fs}$ and a total propagation time of $35 \mathrm{fs}$ are used. The bond length used for these atoms is taken from Ref. 57: 
TABLE I. The dynamic SHGs for $\mathrm{CO}, \mathrm{HF}, \mathrm{HCl}$, and $\mathrm{LiF}$ (unit: a.u.).

\begin{tabular}{|c|c|c|c|c|c|c|c|c|c|}
\hline & \multicolumn{3}{|c|}{$\omega=1.1653 \mathrm{eV}$} & \multicolumn{3}{|c|}{$\omega=1.785 \mathrm{eV}$} & \multicolumn{3}{|c|}{$\omega=1.9593 \mathrm{eV}$} \\
\hline & $\begin{array}{c}\text { Time } \\
\text { domain }\end{array}$ & $\begin{array}{l}\text { Freq. } \\
\text { domain }\end{array}$ & $\begin{array}{c}\text { Ref. } \\
57\end{array}$ & $\begin{array}{c}\text { Time } \\
\text { domain }\end{array}$ & $\begin{array}{l}\text { Freq. } \\
\text { domain }\end{array}$ & $\begin{array}{c}\text { Ref. } \\
57\end{array}$ & $\begin{array}{c}\text { Time } \\
\text { domain }\end{array}$ & $\begin{array}{l}\text { Freq. } \\
\text { domain }\end{array}$ & $\begin{array}{c}\text { Ref. } \\
57\end{array}$ \\
\hline \multicolumn{10}{|c|}{$\mathrm{CO}$} \\
\hline$\beta_{z z z}$ & 36.04 & 35.98 & 35.99 & 39.74 & 39.64 & 39.65 & 41.21 & 41.10 & 41.09 \\
\hline$\beta_{z x x}$ & 9.63 & 9.61 & 9.79 & 11.32 & 11.30 & 11.50 & 12.02 & 11.99 & 12.20 \\
\hline$\beta_{x z x}$ & 9.30 & 9.23 & 9.41 & 10.45 & 10.34 & 10.55 & 10.93 & 10.81 & 11.02 \\
\hline \multicolumn{10}{|c|}{$\mathrm{HF}$} \\
\hline$\beta_{z z z}$ & -11.01 & -11.00 & -11.15 & -11.75 & -11.74 & -11.88 & -12.03 & -12.02 & -12.16 \\
\hline$\beta_{z x x}$ & -2.204 & -2.200 & -2.41 & -2.369 & -2.364 & -2.58 & -2.433 & -2.426 & -2.65 \\
\hline$\beta_{x z x}$ & -2.385 & -2.371 & -2.56 & -2.870 & -2.849 & -3.03 & -3.079 & -3.053 & -3.23 \\
\hline \multicolumn{10}{|c|}{$\mathrm{HCl}$} \\
\hline$\beta_{z z z}$ & -15.08 & -15.04 & -16.67 & -16.73 & -16.67 & -18.46 & -17.39 & -17.32 & -19.17 \\
\hline$\beta_{z x x}$ & -2.349 & -2.336 & -2.31 & -2.740 & -2.716 & -2.65 & -2.919 & -2.876 & -2.80 \\
\hline$\beta_{x z x}$ & -2.824 & -2.773 & -2.76 & -4.222 & -4.132 & -4.10 & -4.990 & -4.813 & -4.78 \\
\hline \multicolumn{10}{|c|}{$\mathrm{LiF}$} \\
\hline$\beta_{z z z}$ & -55.67 & -55.38 & -57.36 & -73.07 & -72.81 & -75.11 & -82.91 & -81.66 & -84.07 \\
\hline$\beta_{z x x}$ & -70.51 & -69.93 & -69.07 & -94.31 & -95.34 & -94.32 & -108.5 & -108.2 & -107.06 \\
\hline$\beta_{x z x}$ & -82.85 & -79.80 & -78.85 & -144.9 & -147.3 & -145.6 & -108.5 & -200.0 & -197.3 \\
\hline
\end{tabular}

$1.1283 \AA$ for $\mathrm{CO}, 1.56386 \AA$ for $\mathrm{LiF}, 0.917 \AA$ for $\mathrm{HF}$, and $1.2746 \AA$ for $\mathrm{HCl}$. The dynamic hyperpolarizabilities are calculated at the frequencies of $1.1653,1.785$, and $1.9593 \mathrm{eV}$. The calculated dynamic SHGs are listed in Table I and ORs are listed in Table II. It can be seen from the table that the dynamic hyperpolarizabilities calculated with time-domain TDDFT and frequency-domain TDDFT agree quite well with each other in most cases. The accuracy of the time-domain method relies also on the error of the spectra analysis. The difference is a little larger for $\mathrm{LiF}$ than for other molecules. This is because the hyperpolarizabilities of LiF are pretty large so that higher order effects have some contributions to the second-order signal. To achieve better results, a weaker electric field should be applied. The difference between the

TABLE II. The dynamic ORs for $\mathrm{CO}, \mathrm{HF}, \mathrm{HCl}$, and $\mathrm{LiF}$ (unit: a.u.).

\begin{tabular}{|c|c|c|c|c|c|c|c|}
\hline & \multicolumn{2}{|c|}{$\omega=1.1653 \mathrm{eV}$} & \multicolumn{3}{|c|}{$\omega=1.785 \mathrm{eV}$} & \multicolumn{2}{|c|}{$\omega=1.9593 \mathrm{eV}$} \\
\hline & $\begin{array}{c}\text { Time } \\
\text { domain }\end{array}$ & $\begin{array}{l}\text { Freq. } \\
\text { domain }\end{array}$ & $\begin{array}{c}\text { Time } \\
\text { domain }\end{array}$ & $\begin{array}{l}\text { Freq. } \\
\text { domain }\end{array}$ & $\begin{array}{c}\text { Ref. } \\
8\end{array}$ & $\begin{array}{c}\text { Time } \\
\text { domain }\end{array}$ & $\begin{array}{c}\text { Freq. } \\
\text { domain }\end{array}$ \\
\hline \multicolumn{8}{|c|}{$\mathrm{CO}$} \\
\hline$\beta_{z z z}$ & 34.39 & 34.36 & 35.47 & 35.43 & & 35.85 & 35.83 \\
\hline$\beta_{z x x}$ & 8.74 & 8.72 & 8.98 & 8.97 & & 9.09 & 9.06 \\
\hline$\beta_{x z x}$ & 8.89 & 8.84 & 9.36 & 9.27 & & 9.46 & 9.43 \\
\hline$\beta_{\|}$ & 31.24 & 31.18 & 32.36 & 32.29 & 32.3 & 32.71 & 32.67 \\
\hline \multicolumn{8}{|c|}{$\mathrm{HF}$} \\
\hline$\beta_{z z z}$ & -10.66 & -10.66 & -10.89 & -10.89 & & -10.98 & -10.97 \\
\hline$\beta_{z x x}$ & -2.20 & -2.19 & -2.35 & -2.35 & & -2.42 & -2.41 \\
\hline$\beta_{x z x}$ & -2.14 & -2.14 & -2.24 & -2.22 & & -2.27 & -2.25 \\
\hline$\beta_{\|}$ & -8.99 & -8.98 & -9.27 & -9.25 & -9.68 & -9.37 & -9.35 \\
\hline \multicolumn{8}{|c|}{$\mathrm{HCl}$} \\
\hline$\beta_{z z z}$ & -14.28 & -14.32 & -14.77 & -14.80 & & -14.90 & -14.98 \\
\hline$\beta_{z x x}$ & -2.34 & -2.35 & -2.67 & -2.72 & & -2.81 & -2.87 \\
\hline$\beta_{x z x}$ & -2.48 & -2.22 & -2.40 & -2.39 & & -2.48 & -2.46 \\
\hline$\beta_{\|}$ & -11.49 & -11.31 & -11.85 & -11.88 & & -12.05 & -12.10 \\
\hline \multicolumn{8}{|c|}{$\mathrm{LiF}$} \\
\hline$\beta_{z z z}$ & -49.31 & -49.22 & -53.67 & -53.23 & & -55.30 & -54.80 \\
\hline$\beta_{z x x}$ & -64.64 & -64.28 & -77.04 & -76.15 & & -83.96 & -81.25 \\
\hline$\beta_{x z x}$ & -63.01 & -61.85 & -71.43 & -69.06 & & -75.36 & -71.97 \\
\hline$\beta_{\|}$ & -105.8 & -104.7 & -120.2 & -117.6 & & -127.1 & -123.0 \\
\hline
\end{tabular}




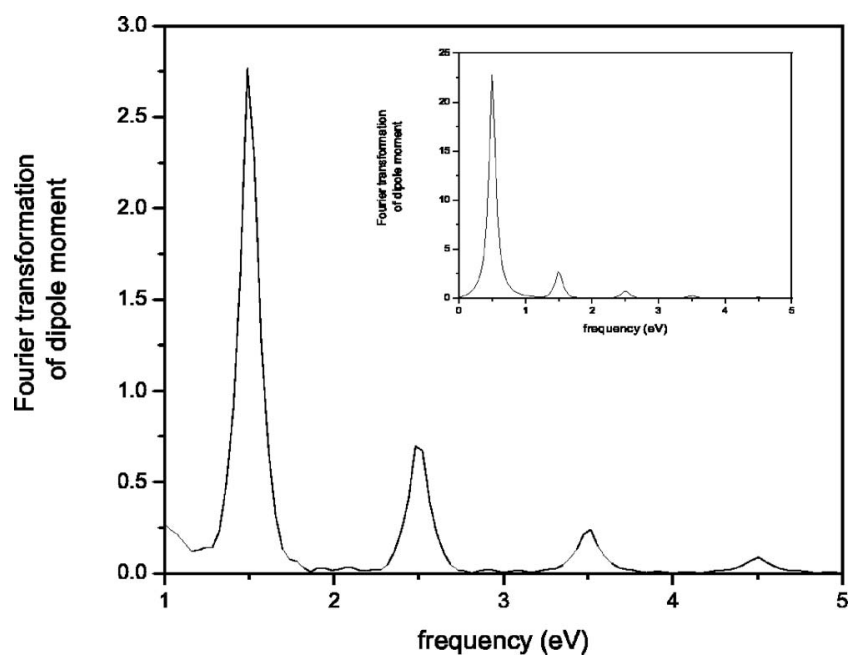

FIG. 4. The Fourier transformation of the time-dependent dipole moment for $\mathrm{Be}$ due to an external field strength of $10 \mathrm{eV} / \mathrm{bohr}$ with frequency of $0.5 \mathrm{eV}$ between 1 and $5 \mathrm{eV}$. (The frequency range of the inset is from 0 to $5 \mathrm{eV}$.)

present results and those of Ref. 57 is mainly due to the differences in the basis set, implementations, and computational details.

The computational effort of time-domain TDDFT is much larger than that of frequency-domain TDDFT for the calculation of dynamic hyperpolarizabilities. However, the time-domain TDDFT is much easier in implementation than the frequency-domain TDDFT because the calculation of the derivatives of $\mathrm{XC}$ potential with respect to the electron density is not required. The time-domain TDDFT can be used to calibrate the implementation of the frequency-domain TDDFT. Furthermore, higher order dynamic hyperpolarizabilities can also be obtained using the time-domain TDDFT. To show this, we solve Eq. (1) for Be atom with a DZVP basis. ${ }^{58}$ The electric field has a strength of $10 \mathrm{eV} / \mathrm{bohr}$ and a frequency of $0.5 \mathrm{eV}$. To avoid the nonadiabatic effects, the external field is fully turned on at the end of the third cycle. To achieve high accuracy, Eq. (1) is solved using the fifthorder Runge-Kutta method with a time step of $0.5 \times 10^{-3}$ fs and a total propagation propagation time of $100 \mathrm{fs}$. The Fourier transformation on the time-dependent dipole moment with a $0.04 \mathrm{eV}$ dephasing is plotted in Fig. 4. The peaks at $1.52 .5,3.5$, and $4.5 \mathrm{eV}$ are significant from this figure. These peaks correspond to the third, fifth, seventh, and ninth harmonic generations, respectively. It is very difficult to estimate higher harmonic generations than the third in frequency domain using perturbation method. However, with time-domain TDDFT this can be done readily. Recently, similar techniques were used to calculate the high harmonic generations in real time domain. ${ }^{59-61}$

\section{CONCLUSION}

In the present work we developed density matrix based TDDFT method in both real time domain and frequency domain to calculate the dynamic hyperpolarizabilities. In real time domain, the TDKS equation is solved for the timedependent density matrix. If the external field is weak, the dynamic polarizabilities in the entire frequency range can readily be obtained. With a time-dependent external electric field oscillating at a certain frequency $\omega$, the dynamic first hyperpolarizability correspond to the $2 \omega$ component of timedependent dipole moment. Dynamic hyperpolarizabilities due to two different frequencies can be calculated similarly. To obtain accurate dynamic hyperpolarizabilities, we find that the strength of the external field should be neither too strong for it results in significant higher order effects nor too weak for the resulting signals are too weak to be identified. Furthermore, this external field should be turned on slowly to prevent the nonadiabatic effects. The expression for the calculation of dynamic hyperpolarizabilities in frequency domain is proposed within the density matrix based TDDFT, and the $2 n+1$ rule for the first hyperpolarizability is easily derived. Similar strategy can also be applied to derive the expression for the higher hyperpolarizabilities. The expressions for the TPA cross section and the transition dipole moment between excited states based on the $2 n+1$ rule are also presented.

The calculated dynamic hyperpolarizabilities based on these two methods are shown to agree very well with each other. The time-domain calculation is more time consuming and not straightforward in the calculation of individual spatial components of higher order hyperpolarizabilities. The frequency-domain method is thus recommended for routine calculations of dynamic hyperpolarizabilities. On the other hand, the time-domain method is easy to implement and can be used to calibrate the implementation of the frequencydomain method. Besides the second-order properties, the time-domain TDDFT method can be easily adopted to calculate much higher order properties which are very difficult to calculate in the frequency domain. The derivatives of the $\mathrm{XC}$ potential with respect to electron density are not evaluated explicitly in the time-domain method, and this facilitates its application to much more complicated forms of the XC potential. Furthermore, the calculation of hyperpolarizabilities in frequency domain relies on a perturbation expansion due to external perturbation and this expansion fails at resonant frequencies. On the other hand, the time-domain formulation is free from such difficulties. It is worth noting that the present method can also be applied to calculate dynamic hyperpolarizabilities within TDHF in both real time domain and frequency domain except that Eqs. (23) and (29) cannot be used since the matrices $A$ and $B$ in Eqs. (18) and (27) do not have the simple relation such as Eq. (19). In addition, for TDHF the high order derivatives of the exchange potential are not needed, and this facilitates its implementation in frequency domain.

\section{ACKNOWLEDGMENTS}

The authors thank Dr. Jingfang Huang for helpful discussions and V. A. Mandelshtam for offering us the filter diagonalization code. Support from Hong Kong Research Grant Council (HKU 7013/05P and G_HK 029/04) is gratefully acknowledged.

${ }^{1}$ D. P. Shelton and J. E. Rice, Chem. Rev. (Washington, D.C.) 94, 3 (1994).

${ }^{2}$ J. L. Brédas, C. Adant, P. Tackx, and A. Persoons, Chem. Rev. (Wash- 
ington, D.C.) 94, 195 (1994).

${ }^{3}$ D. R. Kanis, M. A. Ratner, and T. J. Marks, Chem. Rev. (Washington, D.C.) 94, 195 (1994)

${ }^{4}$ H. A. Kurtz and D. S. Dubis, Rev. Comput. Chem. 12, 241 (1998).

${ }^{5}$ D. Lu, G. H. Chen, J. W. Perry, and W. A. Goddard III, J. Am. Chem. Soc. 116, 10679 (1994); G. H. Chen, D. Lu, and W. A. Goddard III, J. Chem. Phys. 101, 5864 (1994); D. Lu, G. H. Chen, and W. A. Goddard, ibid. 101, 4920 (1994); G. H. Chen and S. Mukamel, J. Phys. Chem. 100, 11080 (1996); J. Chem. Phys. 103, 9355 (1995); J. Am. Chem. Soc. 117, 4945 (1995); W. Z. Liang, S. Yokojima, and G. H. Chen, J. Chem. Phys. 113, 1403 (2000)

${ }^{6}$ E. K. U. Gross and W. Kohn, Adv. Quantum Chem. 21, 255 (1990).

${ }^{7}$ S. J. A. van Gisbergen, J. G. Snijders, and E. J. Baerends, J. Chem. Phys. 109, 10644 (1998).

${ }^{8}$ S. J. A. van Gisbergen, J. G. Snijders, and E. J. Baerends, J. Chem. Phys. 109, 10657 (1998).

${ }^{9}$ J.-I. Iwata, K. Yabana, and G. F. Bertsch, J. Chem. Phys. 115, 8773 (2001).

${ }^{10}$ H. H. Heinze, F. D. Salla, and A. Görling, J. Chem. Phys. 116, 9624 (2002).

${ }^{11}$ P. Salek, O. Vahtras, T. Helgaker, and H. Ågren, J. Chem. Phys. 117, 9630 (2002).

${ }^{12}$ M. Krykunov and J. Autschbach, J. Chem. Phys. 126, 024101 (2007).

${ }^{13}$ X. Andrade, S. Botti, M. A. L. Marques, and A. Rubio, J. Chem. Phys. 126, 184106 (2007).

${ }^{14}$ J. C. Slater, Quantum Theory of Molecules and Solids (McGraw-Hill, New York, 1974), Vol. 4.

${ }^{15}$ S. H. Vosko, L. Wilk, and M. Nusair, Can. J. Phys. 58, 1200 (1980).

${ }^{16}$ M. Grüning, O. V. Gritsenko, S. J. A. van Gisbergen, and E. J. Baerends, J. Chem. Phys. 116, 9591 (2002).

${ }^{17}$ F. A. Bulat, A. Toro-Labbé, B. Champagne, B. Kirtman, and W. Yang, J. Chem. Phys. 123, 014319 (2005).

${ }^{18}$ H. Sekino, Y. Maeda, M. Kamiya, and K. Hirao, J. Chem. Phys. 126, 014107 (2007).

${ }^{19}$ S. Yokojima and G. H. Chen, Phys. Rev. B 59, 7259 (1999).

${ }^{20}$ S. Yokojima and G. H. Chen, Chem. Phys. Lett. 292, 379 (1998)

${ }^{21}$ W. Z. Liang, S. Yokojima, and G. H. Chen, J. Chem. Phys. 110, 1844 (1999); J. Phys. Chem. A 104, 2445 (2000).

${ }^{22}$ C. Y. Yam, S. Yokojima, and G. H. Chen, Phys. Rev. B 68, 153105 (2003).

${ }^{23}$ C. Y. Yam, S. Yokojima, and G. H. Chen, J. Chem. Phys. 119, 8794 (2003)

${ }^{24}$ F. Wang, C. Y. Yam, G. H. Chen, and K. Fan, J. Chem. Phys. 126, 134104 (2007).

${ }^{25}$ See Special Topic Issue, J. Chem. Phys. 123, Issue 6 (2005).

${ }^{26}$ A. Görling, Phys. Rev. Lett. 83, 5459 (1999).

${ }^{27}$ S. Ivanov, S. Hirata, and R. J. Bartlett, Phys. Rev. Lett. 84, 5455 (1999).

${ }^{28}$ W. Yang and Q. Wu, Phys. Rev. Lett. 89, 143002 (2002).

${ }^{29}$ F. Furche, J. Chem. Phys. 114, 5982 (2001).
${ }^{30}$ S. Tretiak and S. Mukamel, Chem. Rev. (Washington, D.C.) 102, 3171 (2002).

${ }^{31}$ S. Tretiak and V. Chernyak, J. Chem. Phys. 119, 8809 (2003).

${ }^{32}$ S. P. Karna and M. Dupuis, J. Comput. Chem. 12, 487 (1991).

${ }^{33}$ X. Li, S. M. Smith, A. N. Markevitch, D. A. Romanov, R. J. Levis, and H. B. Schlegel, Phys. Chem. Chem. Phys. 7, 233 (2005).

${ }^{34}$ A. Castro, M. L. Marques, and A. Rubio, J. Chem. Phys. 121, 3425 (2004).

${ }^{35}$ M. Hochbruck and C. Lubich, SIAM (Soc. Ind. Appl. Math.) J. Numer. Anal. 41, 945 (2003)

${ }^{36}$ W. H. Press, S. A. Teukolsky, W. T. Vetterling, and B. P. Flannery, in Numerical Recipes in FORTRAN 77, 2nd ed. (Cambridge University Press, Cambridge, 1992).

${ }^{37}$ R. Baer, Y. Kurzweil, and L. S. Cederbaum, Isr. J. Chem. 45, 161 (2004).

${ }^{38}$ R. Baer, Phys. Rev. A 62, 063810 (2000).

${ }^{39}$ J. Huang, J. Jia, and M. Minion, J. Comput. Phys. 221, 739 (2007).

${ }^{40}$ R. Baer and D. Neuhauser, J. Chem. Phys. 121, 9803 (2004).

${ }^{41}$ R. Kosloff, J. Phys. Chem. 92, 2087 (1988).

${ }^{42}$ M. R. Wall and D. Neuhauser, J. Chem. Phys. 102, 8011 (1995).

${ }^{43}$ V. A. Mandelshtam and H. S. Taylor, J. Chem. Phys. 106, 5085 (1997).

${ }^{44}$ J. W. Pang, T. Dieckman, J. Feigon, and D. Neuhauser, J. Chem. Phys. 108, 8360 (1998).

${ }^{45}$ M. E. Casida, in Recent Advances in Density Functional Methods, edited by D. P. Chong (Singapore, World Scientific, 1995), Vol. 1, p. 155.

${ }^{46}$ R. Bauernschmitt and R. Ahlrichs, Chem. Phys. Lett. 256, 454 (1996).

${ }^{47}$ S. J. A. Gisbergen, J. G. Snijders, and E. J. Baerends, J. Chem. Phys. 103, 9347 (1995)

${ }^{48}$ L. Jensen, J. Autschbach, and G. C. Schatz, J. Chem. Phys. 122, 224115 (2005).

${ }^{49}$ J. Olsen and P. Jørgensen, J. Chem. Phys. 82, 3235 (1985).

${ }^{50}$ B. Jansik, P. Salek, D. Jonsson, O. Vahtras, and H. Ågren, J. Chem. Phys. 122, 054107 (2005).

${ }^{51}$ V. Chernyak and S. Mukamel, J. Chem. Phys. 112, 3572 (2000).

${ }^{52}$ F. Furche and R. Ahlrichs, J. Chem. Phys. 117, 9535 (2002).

${ }^{53}$ G. H. Chen, C. Y. Yam, S. Yokojima, W. Z. Liang, X. J. Wang, F. Wang, and X. Zheng, http://yangtze.hku.hk/LODESTAR/lodestar.php

${ }^{54}$ S. M. Smith, X. Li, A. N. Markevitch, D. A. Romanov, R. J. Levis, and H. B. Schlegel, J. Phys. Chem. A 109, 5176 (2005).

${ }^{55}$ T. H. Dunning, J. Chem. Phys. 90, 1007 (1989).

${ }^{56}$ D. E. Woon and T. H. Dunning, J. Chem. Phys. 98, 1358 (1993)

${ }^{57}$ M. Pecul, Chem. Phys. Lett. 404, 217 (2005).

${ }^{58}$ N. Godbout, D. R. Salahub, J. Andzelm, and E. Wimmer, Can. J. Phys. 70, 560 (1992).

${ }^{59}$ J. Sun, Z. Guo, and W. Liang, Phys. Rev. B 75, 195438 (2007).

${ }^{60}$ A. Castro, M. A. L. Marques, J. A. Alonso, G. F. Bertsch, and A. Rubio, Eur. Phys. J. D 28, 211 (2004).

${ }^{61}$ S. Erhard and G. K. U. Gross, in Multiphoton Process 1996, edited by P. Lambropoulos and H. Walther (IOP, Bristol, 1997), pp. 37-46. 